OPEN ACCESS

Edited by:

Vito Trianni,

Istituto di Scienze e Tecnologie della Cognizione (ISTC)-CNR, Italy

Reviewed by:

Athanasios Voulodimos, National Technical University of Athens, Greece

Bertrand Collignon,

Eidgenössische Technische Hochschule Lausanne, Switzerland

${ }^{*}$ Correspondence: Tim Landgraf tim.landgraf@fu-berlin.de

Specialty section: This article was submitted to Computational Intelligence, a section of the journal Frontiers in Robotics and Al

Received: 30 November 2017 Accepted: 16 March 2018 Published: 04 April 2018

Citation:

Boenisch F, Rosemann B, Wild B, Dormagen $D$, Wario $F$ and Landgraf $T$

(2018) Tracking All Members of a Honey Bee Colony Over Their Lifetime

Using Learned Models of Correspondence.

Front. Robot. Al 5:35 doi: 10.3389/frobt.2018.00035

\section{Tracking All Members of a Honey Bee Colony Over Their Lifetime Using Learned Models of Correspondence}

\author{
Franziska Boenisch, Benjamin Rosemann, Benjamin Wild, David Dormagen, \\ Fernando Wario and Tim Landgraf*
}

Dahlem Center for Machine Learning and Robotics, FB Mathematik und Informatik, Freie Universität Berlin, Berlin, Germany

Computational approaches to the analysis of collective behavior in social insects increasingly rely on motion paths as an intermediate data layer from which one can infer individual behaviors or social interactions. Honey bees are a popular model for learning and memory. Previous experience has been shown to affect and modulate future social interactions. So far, no lifetime history observations have been reported for all bees of a colony. In a previous work we introduced a recording setup customized to track up to 4,000 marked bees over several weeks. Due to detection and decoding errors of the bee markers, linking the correct correspondences through time is non-trivial. In this contribution we present an in-depth description of the underlying multi-step algorithm which produces motion paths, and also improves the marker decoding accuracy significantly. The proposed solution employs two classifiers to predict the correspondence of two consecutive detections in the first step, and two tracklets in the second. We automatically tracked $\sim 2,000$ marked honey bees over 10 weeks with inexpensive recording hardware using markers without any error correction bits. We found that the proposed two-step tracking reduced incorrect ID decodings from initially $\sim 13 \%$ to around $2 \%$ post-tracking. Alongside this paper, we publish the first trajectory dataset for all bees in a colony, extracted from $\sim 3$ million images covering 3 days. We invite researchers to join the collective scientific effort to investigate this intriguing animal system. All components of our system are open-source.

Keywords: honey bees, Apis mellifera, social insects, tracking, trajectory, lifetime history

\section{INTRODUCTION}

Social insect colonies are popular model organisms for self-organization and collective decision making. Devoid of central control, it often appears miraculous how orderly termites build their nests or ant colonies organize their labor. Honey bees are a particularly popular example-they stand out due to a rich repertoire of communication behaviors (von Frisch, 1965; Seeley, 2010) and their highly flexible division of labor (Robinson, 1992; Johnson, 2010). A honey bee colony robustly adapts to changing conditions, whether it may be a hole in the hive that needs to be repaired, intruders that need to be fended off, brood that needs to be reared, or food that needs to be found and processed. The colony behavior emerges from interactions of many thousand individuals. The complexity that results from the vast number of individuals is increased by the fact that bees are excellent learners: empirical evidence indicates that personal experience can modulate 
communication behavior (Richter and Waddington, 1993; De Marco and Farina, 2001; Goyret and Farina, 2005; Grüter et al., 2006; Grüter and Farina, 2009; Grüter and Ratnieks, 2011; Balbuena et al., 2012). Especially among foragers, personal experience may be very variable. The various locations a forager visits might be dispersed over large distances (up to several kilometers around the hive) and each site might offer different qualities of food, or even pose threats. Thus, no two individuals share the same history and experiences. Evaluating how personal experience shapes the emergence of collective behavior and how individual information is communicated to and processed by the colony requires robust identification of individual bees over long time periods.

However, insects are particularly hard to distinguish by a human observer. Tracking a bee manually is therefore difficult to realize without marking these animals individually. Furthermore, following more than one individual simultaneously is almost impossible for the human eye. Thus, the video recording must be watched once per individual, which, in the case of a bee hive, might be several hundred or thousand times. Processing long time spans or the observation of many bees is therefore highly infeasible, or is limited to only a small group of animals. Most studies furthermore focused on one focal property, such as certain behaviors or the position of the animal. Over the last decades, various aspects of the social interactions in honey bee colonies have been investigated with remarkable efforts in data collection: Naug (2008) manually followed around 1,000 marked bees in a $1 \mathrm{~h}$ long video to analyze food exchange interactions. Baracchi and Cini (2014) manually extracted the positions of 211 bees once per minute for $10 \mathrm{~h}$ of video data to analyze the colony's proximity network. Biesmeijer and Seeley (2005) observed foraging related behaviors of a total of 120 marked bees over 20 days. Couvillon and coworkers manually decoded over 5,000 waggle dances from video (Couvillon et al., 2014). Research questions requiring multiple properties, many individuals, or long time frames are limited by the costs of manual labor.

In recent years, computer vision software for the automatic identification and tracking of animals has evolved into a popular tool for quantifying behavior (Krause et al., 2013; Dell et al., 2014). Although some focal behaviors might be extracted from the video feed directly (Berman et al., 2014; Wiltschko et al.,
2015; Wario et al., 2017), tracking the position of an animal often suffices to infer its behavioral state (Kabra et al., 2013; Eyjolfsdottir et al., 2016; Blut et al., 2017). Tracking bees within a colony is a particularly challenging task due to dense populations, similar target appearance, frequent occlusions, and a significant portion of the colony frequently leaving the hive. The exploration flights of foragers might take several hours, guard bees might stay outside the entire day to inspect incoming individuals. The observation of individual activity over many weeks, hence, requires robust means for unique identification.

For a system that robustly decodes the identity of a given detection, the tracking task reduces to simply connecting matching IDs. Recently, three marker-based insect tracking systems (Mersch et al., 2013; Crall et al., 2015; Gernat et al., 2018) have been proposed that use a binary code with up to 26 bits for error correction (Thompson, 1983). The decoding process can reliably detect and correct errors, or, reject a detection that can not be decoded. There are two disadvantages to this approach. First, error correction requires relatively expensive recording equipment (most systems use at least a $20 \mathrm{MP}$ sensor with a high quality lens). Second, detections that could not be decoded can usually not be integrated into the trajectory, effectively reducing the detection accuracy and sample rate.

In contrast to these solutions, we have developed a system called BeesBook that uses much less expensive recording equipment (Wario et al., 2015). Figure 1 shows our recording setup, Figure 2 visualizes the processing steps performed after the recording. Our system localizes tags with a recall of $98 \%$ at 99\% precision and decodes $86 \%$ IDs correctly without relying on error correcting codes (Wild et al., 2018). See Figure 3 for the tag design. Linking detections only based on matching IDs would quickly accumulate errors, long-term trajectories would exhibit gaps or jumps between individuals. Following individuals robustly, thus, requires a more elaborate tracking algorithm.

The field of multiple object tracking has produced numerous solutions to various use-cases such as pedestrian and vehicle tracking (for reviews see Cox, 1993; Wu et al., 2013; Luo et al., 2014; Betke and Wu, 2016). Animals, especially insects, are harder to distinguish and solutions for tracking multiple animals over long time frames are far less numerous (see Dell et al., 2014 for a review on animal tracking). Since our target subjects may
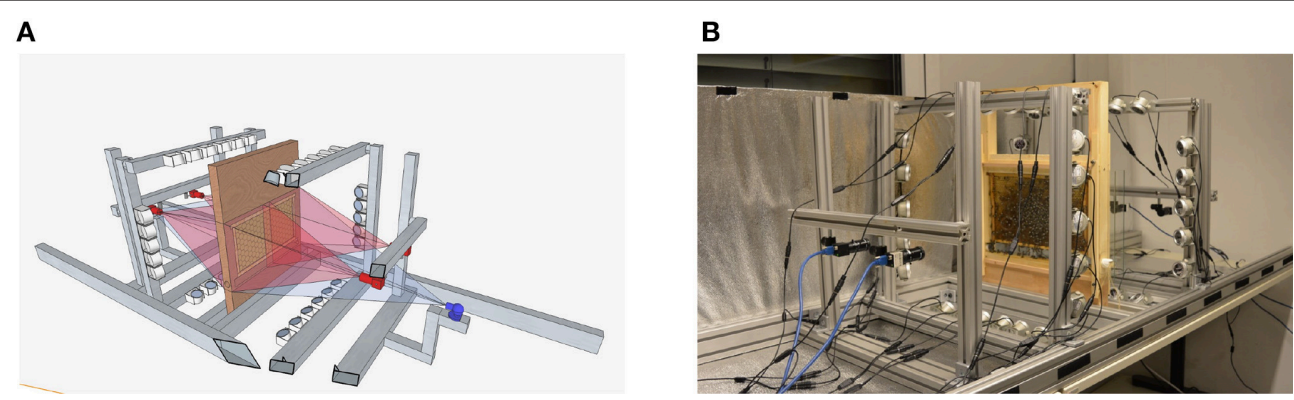

FIGURE 1 | (A) Schematic representation of the setup. Each side of the comb is recorded by two 12 MP PointGray Flea3 cameras. The pictures have an overlap of several centimeters on each side. (B) The recording-setup used in summer 2015. The comb, cameras, and the infrared lights are depicted, the tube that can be used by the bees to leave the setup is not visible. During recording, the setup is covered. Figures adapted from Wario et al. (2015). 


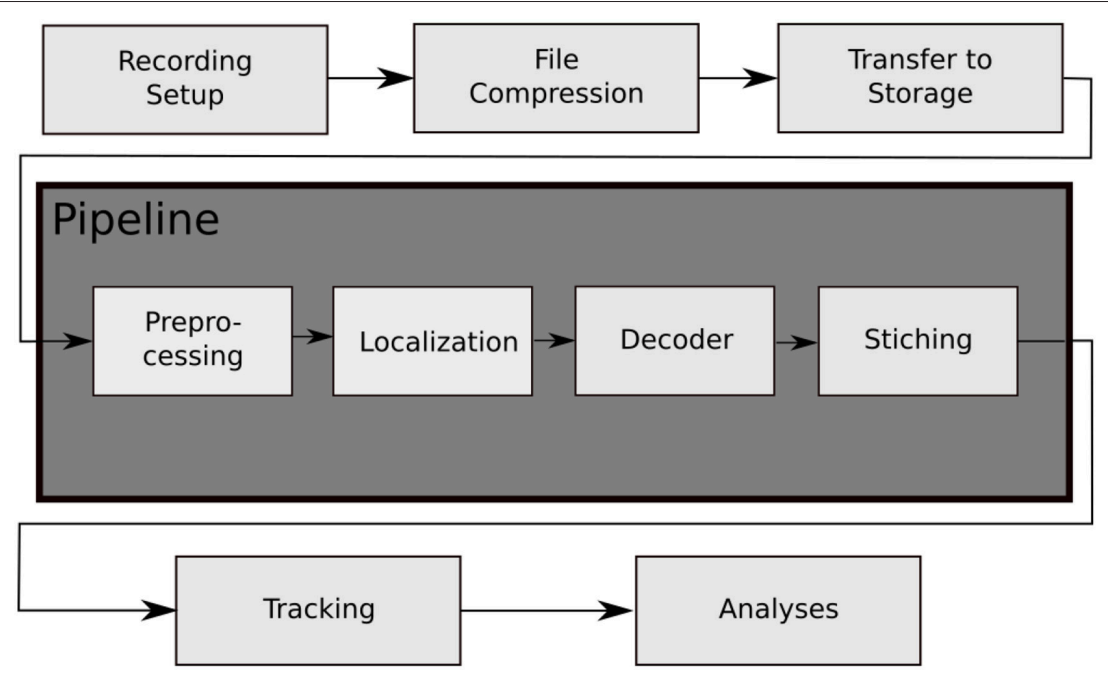

FIGURE 2 | The data processing steps of the BeesBook project. The images captured by the recording setup are compressed on-the-fly to videos containing 1,024 frames each. The video data is then transferred to a large storage from where it can be accessed by the pipeline for processing. Preprocessing: histogram equalization and subsampling for the localizer. Localization: bee markers are localized using a convolutional neural network. Decoding: a second network decodes the IDs and rotation angles. Stitching: the image coordinates of the tags are transformed to hive coordinates and duplicate data in regions where images overlap are removed.
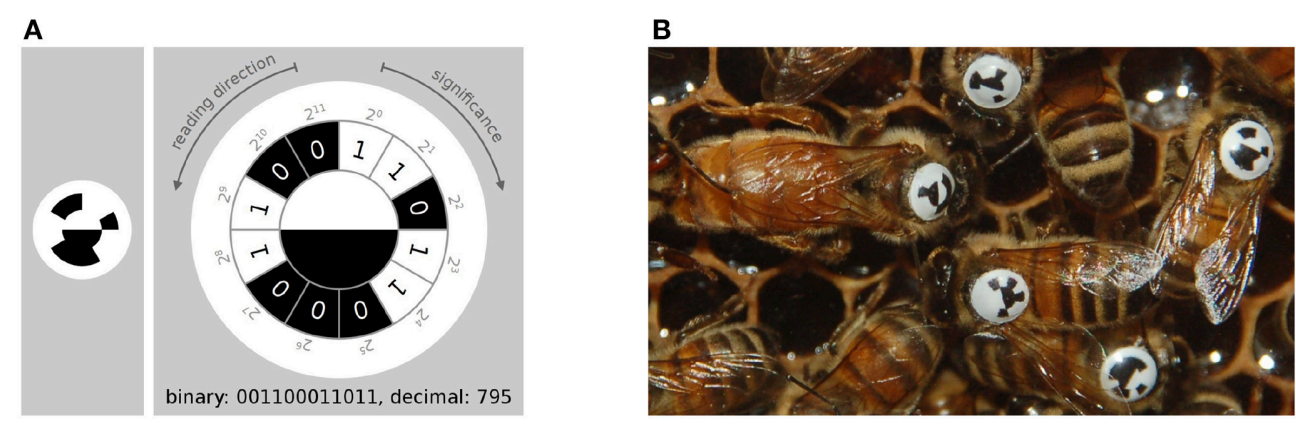

FIGURE 3 | (A) The tag-design in the BeesBook project uses 12 coding segments arranged in an arc around two semi-circles that encode the orientation of the bee. The tag is glued onto the thorax such that the white semi-circle is rotated toward the bee's head. Figure adapted from Wario (2017). (B) Several tagged honey bees on a comb. The round and curved tags are designed to endure heavy duty activities such as cell inspections and foraging trips.

leave the area under observation at any time, the animal's identity cannot be preserved by tracking alone. We require some means of identification for a new detection, whether it be paint marks or number tags on the animals, or identity-preserving descriptors extracted from the detection.

While color codes are infeasible with monochromatic imaging, using image statistics to fingerprint sequences of visible animals (Kühl and Burghardt, 2013; Wang and Yeung, 2013; Pérez-Escudero et al., 2014) may work even with unstructured paint markers. Merging tracklets after occlusions can then be done by matching fingerprints. However, it remains untested whether these approaches can resolve the numerous ambiguities in long-term observations of many hundreds or thousands of bees that may leave the hive for several hours.

In the following, we describe the features that we used to train machine learning classifiers to link individual detections and short tracklets in a crowded bee hive. We evaluate our results with respect to path and ID correctness. We conclude that long-term tracking can be performed without marker-based error correction codes. Tracking can, thus, be conducted without expensive high-resolution, low-noise camera equipment. Instead, decoding errors in simple markers can be mitigated by the proposed tracking solution, leading to a higher final accuracy of the assigned IDs compared to other marker-based systems that do not employ a tracking step.

\section{DESCRIPTION OF METHODS}

\subsection{Problem Statement and Overview of Tracking Approach}

The tracking problem is defined as follows: Given a set of detections (timestamp, location, orientation, and ID information), find correct correspondences among detections over time (tracks) and assign the correct ID to each track. The ID information of the detections can contain errors. Additionally, 
correct correspondences between detections of consecutive frames might not exist due to missing detections caused by occluded markers. In our dataset, the ID information consists of a number in the range of 0 to 4,095, represented by 12 bits. Each bit is given as a value between 0.0 and 1.0 which corresponds to the probability that the bit is set.

To solve the described tracking problem, we propose an iterative tracking approach, similar to previous works (for reviews, see Luo et al., 2014; Betke and $\mathrm{Wu}, 2016)$. We use two steps: 1. Consecutive detections are combined into short but reliable tracklets (Rosemann, 2017). 2. These tracklets are connected over longer gaps (Boenisch, 2017). Previous work employing machine learning mostly scored different distance measures separately to combine them into one thresholded value for the first tracking step (Wu and Nevatia, 2007; Huang et al., 2008; Fasciano et al., 2013; Wang et al., 2014). For merging longer tracks, boosting models to predict a ranking between candidate tracklets have been proposed (Huang et al., 2008; Fasciano et al., 2013). We use machine learning models in both steps to learn the probability that two detections, or tracklets, correspond. We train the models on a manually labeled dataset of ground truth tracklets. The features that are used to predict correspondence can differ between detection level and tracklet level, so we treat these two stages as separate learning problems. Both of our tracking steps use the Hungarian algorithm (Kuhn, 1955) to assign likely matches between detections in subsequent time steps based on the predicted probability of correspondence. In the following, we describe which features are suitable for each step and how we used various regression models to create accurate trajectories. We also explain how we integrate the ID decodings of the markers along a trajectory to predict the most likely ID for this animal, which can then be used to extract long-term tracks covering the whole lifespan of an individual. See Figure $\mathbf{4}$ for an overview of our approach.

\subsection{Step 1: Linking Consecutive Detections}

The first tracking step considers detections in successive frames. To reduce the number of candidates, we consider only sufficiently close detections (we use approximately 200 pixels, or $12 \mathrm{~mm}$ ).

From these candidate pairs we extract three features:

1. Euclidean distance between the first detection and its potential successor.

2. Angular difference of both detections' orientations on the comb plane.

3. Manhattan distance between both detections' ID probabilities.

We use our manually labeled training data to create samples with these features that include both correct and incorrect examples of correspondence. A support vector machine (SVM) with a linear kernel (Cortes and Vapnik, 1995) is then trained on these samples. We also evaluated the performance of a random forest classifier (Ho, 1995) with comparable results. We use the SVM implemented in the scikit-learn library (Pedregosa et al., 2011). Their implementation of the probability estimate uses Platt's method (Platt, 1999). This SVM can then be used get the probability of correspondence for pairs of detections that were not included in the training data. To create short tracks

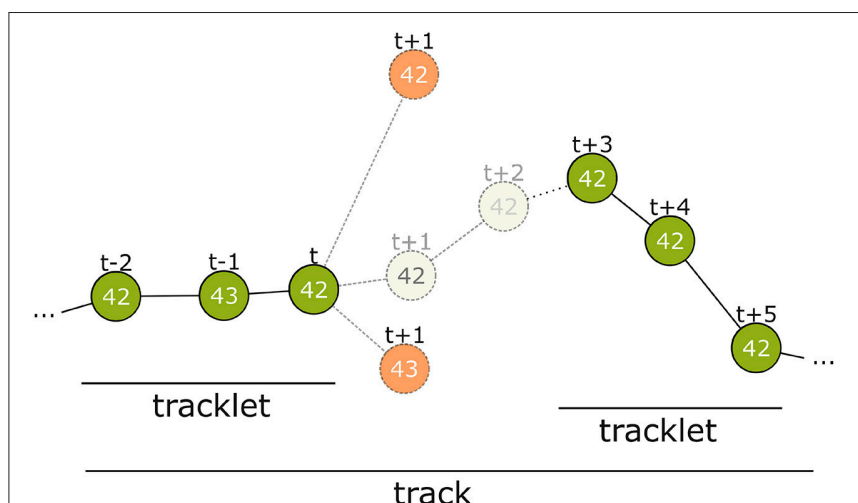

FIGURE 4 | Overview of the tracking process. The first step connects detections from successive frames to tracklets without gaps. At time step $t$ only detections within a certain distance are considered. Even if a candidate has the same ID (top-most candidate with ID 42) it can be disregarded. The correct candidate may be detected with an erroneous ID (see t-1) or may even not be detected at all by the computer vision process. There may be close incorrect candidates that have to be rejected (candidate with ID 43 at $t+1$ ). The model assigns a correspondence probability to all the candidates. If none of them receive a sufficient score the tracklet is closed. In time step $t+3$ a new detection with ID 42 occurs again and is extended into a second tracklet. In tracking step 2, these tracklets are combined to a larger tracklet or track.

(tracklets), we iterate through the recorded data frame by frame and keep a list of open tracklets. Initially, we have one open tracklet for each detection of the first frame. For every time step, we use the SVM to score all new candidates against the last detection of each open tracklet. The Hungarian algorithm is then used to assign the candidate detections to the open tracklets. Tracklets are closed and not further expanded if their best candidate has a probability lower than 0.5 . Detections that could not be assigned to an existing open tracklet are used to begin a new open tracklet that can be expanded in the next time step.

\subsection{Step 2: Merging Tracklets}

The first step yields a set of short tracklets that do not contain gaps and that could be connected with a high confidence. The second tracking step merges these tracklets into longer tracks that can contain gaps of variable duration (for distributions of tracklet and gap length in our data see section 3). Note that a tracklet could consist of a single detection or that its corresponding consecutive tracklet could still begin in the next time step without a gap. To reduce computational complexity we define a maximum gap length of 14 time steps $(\sim 4 \mathrm{~s}$ in our recordings).

Similar to the first tracking step, we use the ground truth dataset to create training samples for a machine learning classifier. We create positive samples (i.e., fragments that should be classified as belonging together) by splitting each manually labeled track once at each time step. Negative samples are generated from each pair of tracks with different IDs which overlapped in time with a maximum gap size of 14 . These are also split at all possible time steps. To include both more positive samples and more short track fragments in the training data, we additionally use every correct sub-track of length 3 or less 
and again split it at all possible locations. This way we generated $1,021,848$ training pairs, $7.4 \%$ of which were positive samples.

In preliminary tests, we found that for the given task of finding correct correspondences between tracklets, a random forest classifier performed best among a selection of classifiers available in scikit-learn (Boenisch, 2017).

Tracklets with two or more detections allow for more complex and discriminative features compared to those used in the first step. For example, matching tracklets separated by longer gaps may require features that reflect a long-term trend (e.g., the direction of motion).

We implemented 31 different features extractable from tracklet pairs. We then used four different feature selection methods from the scikit-learn library to find the features with the highest predictive power. This evaluation was done by splitting the training data further into a smaller training set and validation set. The methods used were Select-K-Best, Recursive Feature Elimination, Recursive Feature Elimination with CrossValidation and the Random Forest Feature Importance for all possible feature subset sizes as provided by scikit-learn (Pedregosa et al., 2011). In all these methods, the same four features (number 1-4 in the listing below) performed best according to the ROC AUC score (Spackman, 1989) that proved to be a suitable metric to measure tracking results. Therefore, we chose them as an initial subset.

We then tried to improve the feature subset manually according to more tracking-specific metrics. The metrics we used were the number of tracks in the ground truth validation set that were reconstructed entirely and correctly, and the number of insertions and deletes in the tracks (for further explanation of the metrics see section 3). We added the features that lead to the highest improvements in these metrics on our validation set. This way, we first added feature 5 and then 6 . After adding feature 6 , the expansion of the subset with any other feature only lead to a performance decrease in form of more insertions and less complete tracks. We therefore kept the following six features. Visualizations of features 2-5 can be found in Figure 5.

1. Manhattan distance of both tracklets' bitwise averaged IDs.

2. Euclidean distance of last detection of tracklet 1 to first detection of tracklet 2.

3. Forward error: Euclidean distance of linear extrapolation of last motion in first tracklet to first detection in second tracklet.

4. Backward error: Euclidean distance of linear extrapolation of first motion in second tracklet to last detection in first tracklet.

5. Angular difference of tag orientation between the last detection of the first tracklet and the first detection of the second tracklet.

6. Difference of confidence: All IDs in both tracklets are averaged with a bitwise median, we select the bit that is closest to 0.5 for each tracklet, calculate the absolute difference to 0.5 (the confidence) and compute the absolute difference of these two confidences.

\subsubsection{Track ID Assignment}

After the second tracking step, we determine the ID of the tracked bee by calculating the median of the bitwise ID probabilities of all detections in the track. The final ID is then determined by binarizing the resulting probabilities for each bit with probability threshold 0.5 .

\subsubsection{Parallelization}

Tracks with a length of several minutes already display a very accurate ID decoding (see section 3). To calculate longer tracks of up to several days and weeks, we execute the tracking step 1 and step 2 for intervals of $1 \mathrm{~h}$ and then merge the results to longer tracks based on the assigned ID. This allows us to effectively parallelize the tracking calculation and track the entire season of
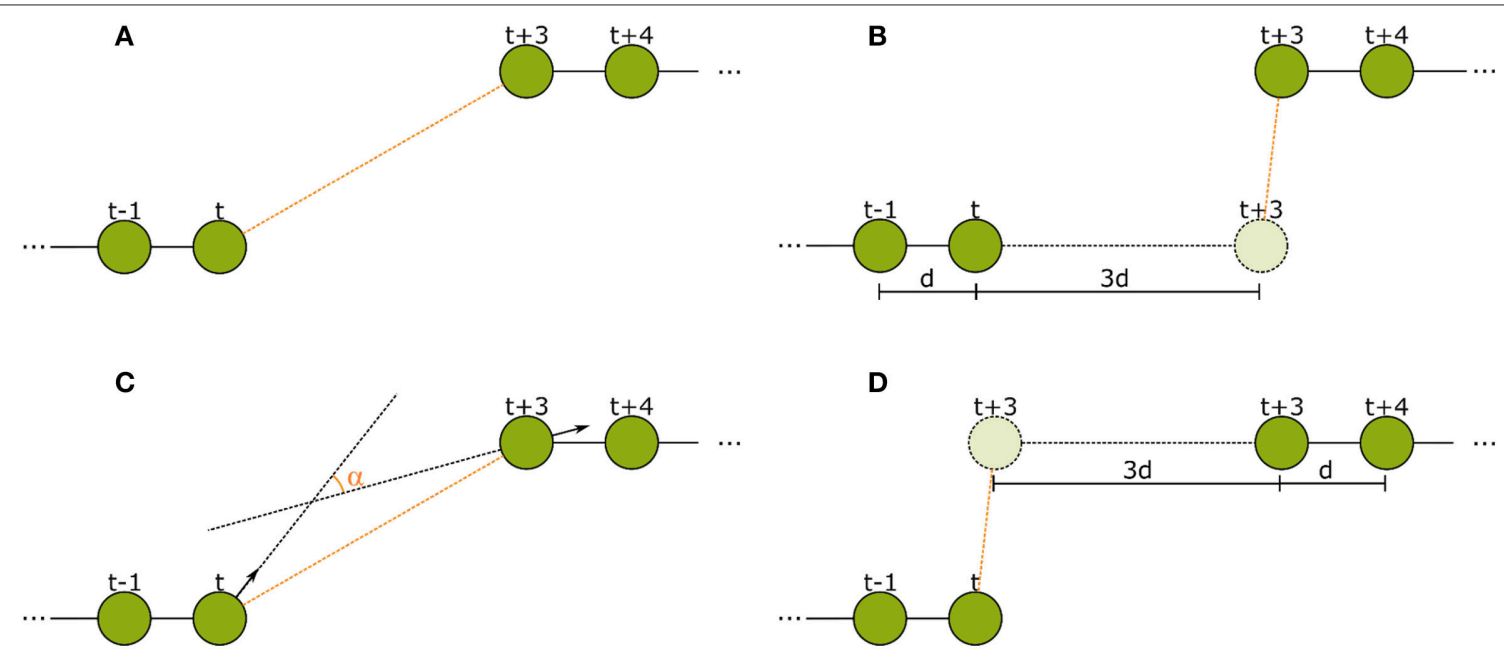

FIGURE 5 | The spatial features used in the second tracking step. (A) Euclidean distance between the last detection of tracklet 1 and the first detection of tracklet 2. (B) Forward error: Euclidean distance of the extrapolation of the last movement vector in tracklet 1 to the first detection in tracklet 2. (C) Angular difference between the tag orientations of the last detection in tracklet 1 and the first detection in tracklet 2. (D) Backward error: Euclidean distance between the reverse extrapolation of the first movement vector of tracklet 2 to the last detection of tracklet 1. 
10 weeks of data in less than a week on a small cluster with $<100$ CPU cores.

\section{RESULTS AND EVALUATION}

We marked an entire colony of 1,953 bees in a 2 days session and continuously added marked young bees that were bred in an incubation chamber. In total, 2,775 bees were marked. The BeesBook system was used to record 10 weeks of continuous image data ( $3 \mathrm{~Hz}$ sample rate) of a one-frame observation hive. The image recordings were stored and processed after the recording season. The computer vision pipeline was executed on a Cray XC30 supercomputer. In total, 3,614,742,669 detections were extracted from $67,972,617$ single frames, corresponding to $16,993,154$ snapshots of the four cameras. Please note that the data could also be processed in real-time using consumer hardware (Wild et al., 2018).

Two ground truth datasets for the training and evaluation of our method were created manually. A custom program was used to mark the positions of an animal and to define its ID (Mischek, 2016). Details on each dataset can be found in Table 1. To avoid overfitting to specific colony states, the datasets were chosen to contain both high activity (around noon) and low activity (in the early morning hours) periods, different cameras and, therefore, different comb areas. Dataset 2015.1 was used to train and validate classifiers and dataset 2015.2 was used to test their performance.

Dataset 2015.1 contains 18,085 detections from which we extracted 36,045 sample pairs (i.e., all pairs with a distance of

TABLE 1 | Dataset 2015.1 was used for training and dataset 2015.2 for testing.

\begin{tabular}{lcc}
\hline Dataset & $\mathbf{2 0 1 5 . 1}$ & $\mathbf{2 0 1 5 . 2}$ \\
\hline Date & 18.09 .2015 & 22.09 .2015 \\
Times & $11: 36 ; 04: 51$ & $13: 36$ \\
Frames & $201(3 \mathrm{fps})$ & $200(3 \mathrm{fps})$ \\
Detections & 18,085 & 10,945 \\
False positives & $222(1.23 \%)$ & $82(0.75 \%)$ \\
Individuals & 144 & 98 \\
\hline
\end{tabular}

The number of detections is the number of tags localized and decoded by the deep learning approach over all frames in the dataset. The number of false positives shows how many times the deep learning pipeline detects a detection when there is none. The number of individuals indicates how many different bees are present in the dataset.
$<200$ pixels in consecutive frames). These samples were used to train the SVM which is used to link consecutive detections together (tracking step 1). Hyperparameters were determined manually using cross-validation on this dataset. The final model was evaluated on dataset 2015.2.

Tracklets for the training and evaluation of a random forest classifier (tracking step 2) were extracted from datasets 2015.1, respectively 2015.2 (see section 2 for details). Hyperparameters were optimized with hyperopt-sklearn (Komer et al., 2014) on dataset 2015.1 and the optimized model was then tested on dataset 2015.2.

To validate the success of the tracking, we analyzed its impact on several metrics in the tracks, namely:

1. ID Improvement

2. Proportion of complete tracks

3. Correctness of resulting tracklets

4. Length of resulting tracklets

To be able to evaluate the improvement through the presented iterative tracking approach, we compare the results of the two tracking steps to the naive approach of linking the original detections over time based on their initial decoded ID only, in the following referred to as "baseline." For an overview on the improvements achieved by the different tracking steps see Table 2 .

\subsection{ID Improvement}

An important goal of the tracking is to correct IDs of detections which could not be decoded correctly by the computer vision system. Without the tracking algorithm described above, all further behavioral analyses would have to consider this substantial proportion of erroneous decodings. In our dataset, $13.3 \%$ of all detections have an incorrectly decoded ID (Wild et al., 2018).

In the ground truth dataset we manually assigned detections that correspond to the same animal to one trajectory. The ground truth data can therefore be considered as the "perfect tracking." Even on these perfect tracks the median ID assignment algorithm described above provides incorrect IDs for $0.6 \%$ of all detections, due to partial occlusions, motion blur and image noise. This represents the lower error bound for the tracking system. As shown in Figure 6, the first tracking step reduces the fraction of incorrect IDs from 13.3 to $3.9 \%$ of all detections. The second step further improves this result to only $1.9 \%$ incorrect IDs.

TABLE 2 | Different metrics were used to compare the two tracking steps to both a naive baseline based on the detection IDs and to manually created tracks without errors (perfect tracking).

\begin{tabular}{|c|c|c|c|c|}
\hline & Baseline (\%) & After step 1 (\%) & After step 2 (\%) & Perfect tracking (\%) \\
\hline Incorrect detection IDs & 13.3 & 3.9 & 1.9 & 0.6 \\
\hline Incorrect track IDs & 63.5 & 27.2 & 18.2 & 8.2 \\
\hline Complete tracks & 10.2 & 26.5 & 70.4 & 77.6 \\
\hline Detections missing from their track (deletions) & 32.2 & 1.38 & 2.37 & 0 \\
\hline Tracks with at least one deletion & 94.6 & 26.7 & 18.25 & 0 \\
\hline
\end{tabular}

In all cases, the baseline performs worst and the two tracking steps successively improve the performance. 
Most errors occur in short tracklets (see Figure 7). Therefore, the $1.9 \%$ erroneous ID assignments correspond to $18.2 \%$ of the resulting tracklets being assigned an incorrect median ID. This is an improvement over the naive baseline and the first tracking step with 63.5 and $27.2 \%$, respectively. A perfect tracking could reduce this to $8.2 \%$ (see Figure 8 ).

\subsection{Proportion of Complete Tracks}

Almost all gaps between detections in our ground truth tracks are no longer than 14 frames (99.76\%, see Figure 9). Even though

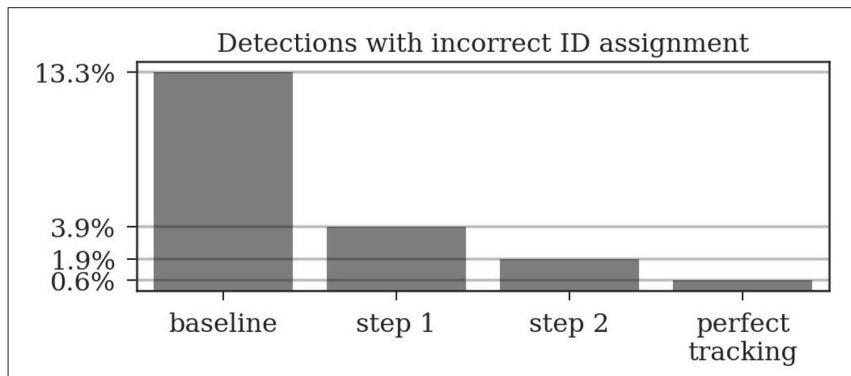

FIGURE 6 | Around $13 \%$ of the raw detections are incorrectly decoded. The first tracking step already reduces this error to around $4 \%$ and the second step further reduces it to around $2 \%$. Even a perfect tracking (defined by the human ground truth) would still result in $0.6 \%$ incorrect IDs when using the proposed ID assignment method.

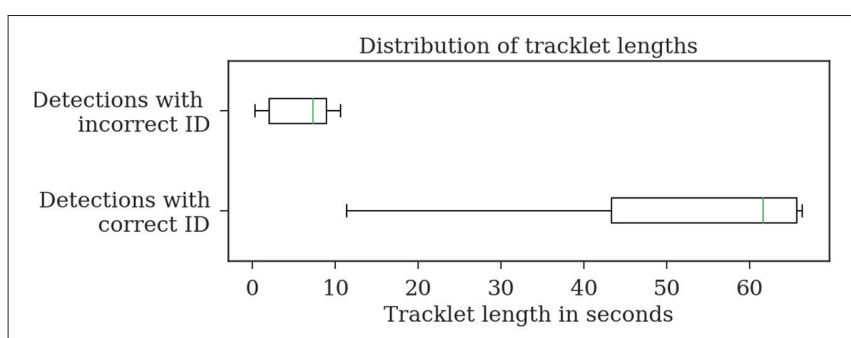

FIGURE 7 | Evaluation of the tracklet lengths of incorrectly assigned detection IDs after the second tracking step reveals that all errors in the test dataset 2015.2 happen in very short tracklets. Note that this dataset covers a duration of around $1 \mathrm{~min}$.

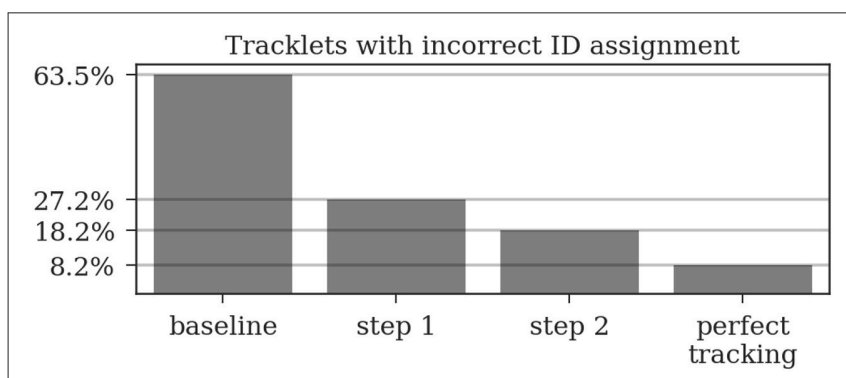

FIGURE 8 | A naive tracking approach using only the detection IDs would result in around $64 \%$ of all tracks being assigned an incorrect ID. Our two-step tracking approach reduces this to around 27 and 18\%, respectively. Due to the short length of most incorrect tracklets, these $18.2 \%$ account for only $1.9 \%$ of the detections. Using our ID assignment method without any tracking errors would reduce the error to $8.2 \%$. large gaps between detections are rare, long tracks are likely to contain at least one such gap: Only around one third (34.7\%) of the ground truth tracks contain no gaps and $77.6 \%$ contain only gaps shorter than 14 frames. As displayed in Figure 10, the baseline tracking finds only $10.2 \%$ complete tracks without errors (i.e., $30 \%$ of all tracks with no gaps). Step 1 is able to correctly assemble $26.5 \%$ complete tracks (i.e., around $76.5 \%$ of all tracks containing no gaps). Step 2 correctly assembles $70.4 \%$ complete tracks (about $90.4 \%$ of all tracks with a maximum gap size of $<14$ frames).

\subsection{Correctness of Resulting Tracklets}

To characterize the type of errors in our tracking results, we define a number of additional metrics. We counted detections that were incorrectly introduced into a track as insertions. Both tracking steps and the baseline inserted only one incorrect detection into another tracklet. Thus, $<1 \%$ of both detections and tracklets were affected.

We counted detections that were missing from a tracklet (and were replaced by a gap) as deletions. In the baseline, $32.2 \%$ of all detections were missing from their corresponding track $(94.6 \%$
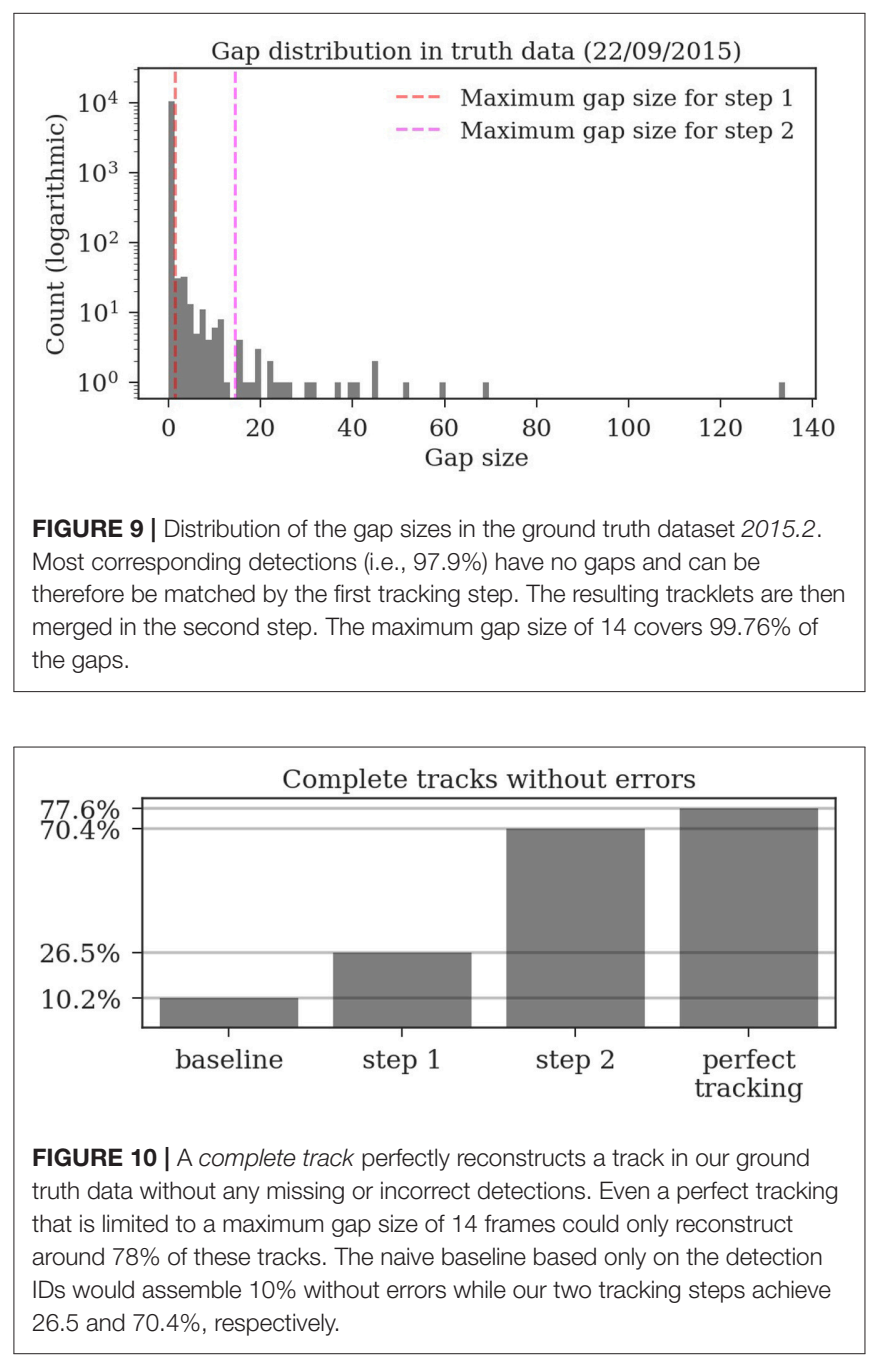


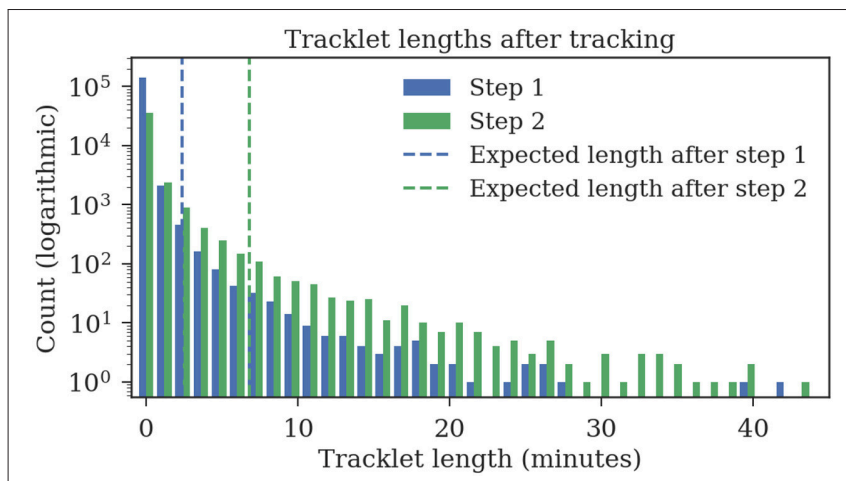

FIGURE 11 | Track lengths after tracking $1 \mathrm{~h}$ of video data at three frames per second. The expected length of a track is 2:23 min after the first step and 6:48 min after the second step.

of all tracks had at least one deletion). After the first step, $1.38 \%$ of detections were missing from their track, affecting $26.7 \%$ of all tracks. After the second step, $2.37 \%$ of all detections and $18.25 \%$ of all tracks were still affected.

We also evaluated whether incorrect detections were contained in a track in situations where the correct detection would have been available (instead of a gap) as mismatches, but no resulting tracks contained such mismatches.

\subsection{Length of Resulting Tracklets}

The ground truth datasets contain only short tracks with a maximum length of $1 \mathrm{~min}$. To evaluate the average length of the tracks, we also tracked $1 \mathrm{~h}$ of data for which no ground truth data was available. The first tracking step yields shorter fragments with an expected length of 2:23 min, the second tracking step merges these fragments to tracklets with an expected duration of 6:48 min (refer to Figure 11 for tracklet length distributions).

\section{DISCUSSION}

We have presented a multi-step tracking algorithm for fragmentary and partially erroneous detections of honey bee markers. We have applied the proposed algorithm to produce long-term trajectories of all honey bees in a colony of approximately 2,000 animals. Our dataset comprises 71 days of continuous positional data at a recording rate of $3 \mathrm{~Hz}$. The presented dataset is by far the most detailed reflection of individual activities of the members of a honey bee colony. The dataset covers the entire lifespan of many hundreds of animals from the day they emerge from their brood cell until the day they die. Honey bees rely on a flexible but generally age-dependent division of labor. Hence, our dataset reflects all essential aspects of a self-sustaining colony, from an egg-laying queen and brood rearing young workers, to food collection, and colony defense. We have released a 3 days sample dataset for the interested reader (Boenisch et al., 2018). Our implementation of the proposed tracking algorithm is available online $e^{1}$.

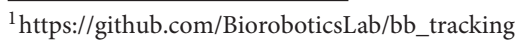

The tracking framework presented in the previous sections is an essential part of the BeesBook system. It provides a computationally efficient approach to determine the correct IDs for more than $98 \%$ of the individuals in the honey bee hive without using extra bits for error correction.

Although it is possible to use error correction with 12 bit markers, this would reduce the number of coding bits and therefore the number of observable animals. While others chose to increase the number of bits on the marker, we solved the problem in the tracking stage. With the proposed system, we were able to reduce hardware costs for cameras and storage. When applied to the raw output of the image decoding step, the accuracy of other systems that use error-correction (for example Mersch et al., 2013) may even be improved further.

Our system provides highly accurate movement paths of bees. Given a long-term observation of several weeks, these paths, however, can still be considered short fragments. Since the IDs of these tracklets are very accurate, they can now be linked by matching IDs only.

Still, some aspects of the system can be improved. To train our classifiers, we need a sufficiently large, manually labeled dataset. Rice et al. (2015) proposed a method to create a similar dataset interactively, reducing the required manual work. Also, the circular coding scheme of our markers causes some bit configurations to appear similar under certain object poses. This knowledge could be integrated into our ID determination algorithm. The IDs along a trajectory might not provide an equal amount of information. Some might be recorded under fast motion and are therefore less reliable. Other detections could have been recorded from a still bee whose tag was partially occluded. Considering similar readings as less informative might improve the ID accuracy of our method. Still, with the proposed method there are only $1.9 \%$ detections incorrectly decoded, mostly in very short tracklets.

The resulting trajectories can now be used for further analyses of individual honey bee behavior or interactions in the social network. In addition to the three day dataset published alongside this paper, we plan to publish two more datasets covering more than 60 days of recordings, each. With this data we can investigate how bees acquire information in the colony and how that experience modulates future behavior and interactions. We hope that through this work we can interest researchers to join the collective effort of investigating the individual and collective intelligence of the honey bee, a model organism that bears a vast number of fascinating research questions.

\section{ETHICS STATEMENT}

German law does not require approval of an ethics committee for studies involving insects.

\section{AUTHOR CONTRIBUTIONS}

BR and TL: Conceptualization; FB, BR, BW, DD, and TL: Methodology; FB and BR: Software; TL: Resources, supervision, 
and project administration; FB, BR, and BW: Data curation; FB, BW, DD, and TL: Writing-original draft; FB, BW, FW, DD, and TL: Writing-review and editing and visualization.

\section{FUNDING}

FW received funding from the German Academic Exchange Service (DAAD). DD received funding from the Andrea von

\section{REFERENCES}

Balbuena, M. S., Molinas, J., and Farina, W. M. (2012). Honeybee recruitment to scented food sources: correlations between in-hive social interactions and foraging decisions. Behav. Ecol. Sociobiol. 66, 445-452. doi: 10.1007/S00265-01 1-1290-3

Baracchi, D., and Cini, A. (2014). A socio-spatial combined approach confirms a highly compartmentalised structure in honeybees. Ethology 120, 1167-1176. doi: $10.1111 /$ eth. 12290

Berman, G. J., Choi, D. M., Bialek, W., and Shaevitz, J. W. (2014). Mapping the stereotyped behaviour of freely moving fruit flies. J. R. Soc. Interface 11:20140672. doi: 10.1098/rsif.2014.0672

Betke, M., and Wu, Z. (2016). Data Association for Multi-Object Visual Tracking. Synthesis Lectures on Computer Vision, ( San Rafael, CA: Morgan \& Claypool Publishers)

Biesmeijer, J. C., and Seeley, T. D. (2005). The use of waggle dance information by honey bees throughout their foraging careers. Behav. Ecol. Sociobiol. 59, 133-142. doi: 10.1007/s00265-005-0019-6

Blut, C., Crespi, A., Mersch, D., Keller, L., Zhao, L., Kollmann, M., et al. (2017). Automated computer-based detection of encounter behaviours in groups of honeybees. Sci. Rep. 7:17663. doi: 10.1038/s41598-017-17863-4

Boenisch, F. (2017). Feature Engineering and Probabilistic Tracking on Honey Bee Trajectories. Bachelor thesis, Freie Universität Berlin.

Boenisch, F., Rosemann, B., Wild, B., Wario, F., Dormagen, D., and Landgraf, T. (2018). BeesBook Recording Season 2015 Sample. Avaialble online at: synapse.org/\#!Synapse:syn11737848

Cortes, C., and Vapnik, V. (1995). Support-vector networks. Mach. Learn. 20, 273-297.

Couvillon, M. J., Schürch, R., and Ratnieks, F. L. W. (2014). Waggle dance distances as integrative indicators of seasonal foraging challenges. PLoS ONE 9:e93495. doi: 10.1371/journal.pone.0093495

Cox, I. J. (1993). A review of statistical data association techniques for motion correspondence. Int. J. Comput. Vis. 10, 53-66.

Crall, J. D., Gravish, N., Mountcastle, A. M., and Combes, S. A. (2015). BEEtag: a low-cost, image-based tracking system for the study of animal behavior and locomotion. PLoS ONE 10:e0136487. doi: 10.1371/journal.pone.0136487

De Marco, R., and Farina, W. (2001). Changes in food source profitability affect the trophallactic and dance behavior of forager honeybees (Apis mellifera L.). Behav. Ecol. Sociobiol. 50, 441-449. doi: 10.1007/s002650100382

Dell, A. I., Bender, J. A., Branson, K., Couzin, I. D., de Polavieja, G. G., Noldus, L. P., et al. (2014). Automated image-based tracking and its application in ecology. Trends Ecol. Evol. 29, 417-428. doi: 10.1016/j.tree.2014.05.004

Eyjolfsdottir, E., Branson, K., Yue, Y., and Perona, P. (2016). Learning recurrent representations for hierarchical behavior modeling. arXiv:1611.00094 [cs].

Fasciano, T., Nguyen, H., Dornhaus, A., and Shin, M. C. (2013). "Tracking multiple ants in a colony," in 2013 IEEE Workshop on Applications of Computer Vision (WACV) (Clearwater Beach, FL), 534-540.

Gernat, T., Rao, V. D., Middendorf, M., Dankowicz, H., Goldenfeld, N., and Robinson, G. E. (2018). Automated monitoring of behavior reveals bursty interaction patterns and rapid spreading dynamics in honeybee social networks. Proc. Natl. Acad. Sci. U.S.A. 115, 1433-1438. doi: $10.1073 /$ pnas. 1713568115

Goyret, J., and Farina, W. M. (2005). Non-random nectar unloading interactions between foragers and their receivers in the honeybee hive. Naturwissenschaften 92, 440-443. doi: 10.1007/s00114-005-0016-7
Braun Foundation. This work was in part funded by the Klaus Tschira Foundation. We also acknowledge the support by the Open Access Publication Initiative of the Freie Universität Berlin.

\section{ACKNOWLEDGMENTS}

We are indebted to the help of Jakob Mischek for his preliminary work and his help with creating the ground truth data.

Grüter, C., Acosta, L. E., and Farina, W. M. (2006). Propagation of olfactory information within the honeybee hive. Behav. Ecol. Sociobiol. 60, 707-715. doi: 10.1007/s00265-006-0214-0

Grüter, C., and Farina, W. M. (2009). Past experiences affect interaction patterns among foragers and hive-mates in honeybees. Ethology 115, 790-797. doi: 10.1111/j.1439-0310.2009.01670.x

Grüter, C., and Ratnieks, F. L. W. (2011). Honeybee foragers increase the use of waggle dance information when private information becomes unrewarding. Anim. Behav. 81, 949-954. doi: 10.1016/j.anbehav.2011. 01.014

Ho, T. K. (1995). "Random decision forests," in Proceedings of the Third International Conference on Document Analysis and Recognition 1995, Vol. 1 (Montreal, QC), 278-282.

Huang, C., Wu, B., and Nevatia, R. (2008). "Robust object tracking by hierarchical association of detection responses," in European Conference on Computer Vision (Marseille: Springer), 788-801.

Johnson, B. R. (2010). Division of labor in honeybees: form, function, and proximate mechanisms. Behav. Ecol. Sociobiol. 64, 305-316. doi: 10.1007/s00265-009-0874-7

Kabra, M., Robie, A. A., Rivera-Alba, M., Branson, S., and Branson, K. (2013). JAABA: interactive machine learning for automatic annotation of animal behavior. Nat. Methods 10, 64-67. doi: 10.1038/ nmeth.2281

Kühl, H. S., and Burghardt, T. (2013). Animal biometrics: quantifying and detecting phenotypic appearance. Trends Ecol. Evol. 28, 432-441. doi: 10.1016/j.tree.2013.02.013

Komer, B., Bergstra, J., and Eliasmith, C. (2014). "Hyperopt-sklearn: automatic hyperparameter configuration for scikit-learn," in ICML Workshop on AutoML (Beijing).

Krause, J., Krause, S., Arlinghaus, R., Psorakis, I., Roberts, S., and Rutz, C. (2013). Reality mining of animal social systems. Trends Ecol. Evol. 28, 541-551. doi: 10.1016/j.tree.2013.06.002

Kuhn, H. W. (1955). The Hungarian method for the assignment problem. Naval Res. Logist. Q. 2, 83-97.

Luo, W., Xing, J., Milan, A., Zhang, X., Liu, W., Zhao, X., et al. (2014). Multiple object tracking: a literature review. arXiv:1409.7618 [cs].

Mersch, D. P., Crespi, A., and Keller, L. (2013). Tracking individuals shows spatial fidelity is a key regulator of ant social organization. Science 340, 1090-1093. doi: $10.1126 /$ science. 1234316

Mischek, J. (2016). Probabilistisches Tracking von Bienenpfaden. Master thesis, Freie Universität Berlin.

Naug, D. (2008). Structure of the social network and its influence on transmission dynamics in a honeybee colony. Behav. Ecol. Sociobiol. 62, 1719-1725. doi: 10.1007/s00265-008-0600-x

Pedregosa, F., Varoquaux, G., Gramfort, A., Michel, V., Thirion, B., Grisel, O., et al. (2011). Scikit-learn: machine learning in Python. J. Mach. Learn. Res. 12, 2825-2830.

Pérez-Escudero, A., Vicente-Page, J., Hinz, R. C., Arganda, S., and de Polavieja, G. G. (2014). idTracker: tracking individuals in a group by automatic identification of unmarked animals. Nat. Methods 11, 743-748. doi: $10.1038 /$ nmeth. 2994

Platt, J. C. (1999). "Probabilistic outputs for support vector machines and comparisons to regularized likelihood methods," in Advances in Large Margin Classifiers, eds A. J. Srnola, P. Bartlett, B. Schélkopf, and D. Schuurrnans (Cambridge, MA: MIT Press), 61-74. 
Rice, L., Dornhausy, A., and Shin, M. C. (2015). "Efficient training of multiple ant tracking," in IEEE Winter Conference on Applications of Computer Vision (Waikoloa, HI), 117-123.

Richter, M. R., and Waddington, K. D. (1993). Past foraging experience influences honey bee dance behaviour. Anim. Behav. 46, 123-128.

Robinson, G. E. (1992). Regulation of division of labor in insect societies. Annu. Rev. Entomol. 37, 637-665.

Rosemann, B. (2017). Ein Erweiterbares System für Experimente Mit Multi-Target Tracking Von Markierten Bienen. Master thesis, Freie Universität Berlin.

Seeley, T. D. (2010). Honeybee Democracy. Princeton, NJ: Princeton University Press.

Spackman, K. A. (1989). "Signal detection theory: valuable tools for evaluating inductive learning," in Proceedings of the Sixth International Workshop on Machine Learning (San Francisco, CA: Morgan Kaufmann Publishers Inc.), 160-163.

Thompson, T. M. (1983). From Error-Correcting Codes Through Sphere Packings to Simple Groups. Cambridge, MA: Cambridge University Press.

von Frisch, K. (1965). Tanzsprache und Orientierung der Bienen. Berlin; Heidelberg: Springer.

Wang, L., Yung, N. H. C., and Xu, L. (2014). Multiple-human tracking by iterative data association and detection update. IEEE Trans. Intell. Transport. Syst. 15, 1886-1899. doi: 10.1109/TITS.2014. 2303196

Wang, N., and Yeung, D.-Y. (2013). "Learning a deep compact image representation for visual tracking," in Proceedings of the 26th International Conference on Neural Information Processing Systems-Vol. 1 (Portland, OR: Curran Associates Inc.), 809-817.

Wario, F. (2017). A Computer Vision Based System for the Automatic Analysis of Social Networks in Honey Bee Colonies. Ph.D. dissertation, Freie Universität Berlin.
Wario, F., Wild, B., Couvillon, M. J., Rojas, R., and Landgraf, T. (2015). Automatic methods for long-term tracking and the detection and decoding of communication dances in honeybees. Front. Ecol. Evol. 3:103. doi: $10.3389 /$ fevo.2015.00103

Wario, F., Wild, B., Rojas, R., and Landgraf, T. (2017). Automatic detection and decoding of honey bee waggle dances. arXiv preprint arXiv:1708.06590.

Wild, B., Sixt, L., and Landgraf, T. (2018). Automatic localization and decoding of honeybee markers using deep convolutional neural networks. arXiv:1802.04557 [cs].

Wiltschko, A., Johnson, M., Iurilli, G., Peterson, R., Katon, J., Pashkovski, S., et al. (2015). Mapping sub-second structure in mouse behavior. Neuron 88, 1121-1135. doi: 10.1016/j.neuron.2015.11.031

Wu, B., and Nevatia, R. (2007). Detection and tracking of multiple, partially occluded humans by Bayesian combination of edgelet based part detectors. Int. J. Comput. Vis. 75, 247-266. doi: 10.1007/s11263-006-0027-7

Wu, Y., Lim, J., and Yang, M. H. (2013). "Online object tracking: a benchmark," in 2013 IEEE Conference on Computer Vision and Pattern Recognition (Portland, OR) 2411-2418.

Conflict of Interest Statement: The authors declare that the research was conducted in the absence of any commercial or financial relationships that could be construed as a potential conflict of interest.

Copyright (๑) 2018 Boenisch, Rosemann, Wild, Dormagen, Wario and Landgraf. This is an open-access article distributed under the terms of the Creative Commons Attribution License (CC BY). The use, distribution or reproduction in other forums is permitted, provided the original author(s) and the copyright owner are credited and that the original publication in this journal is cited, in accordance with accepted academic practice. No use, distribution or reproduction is permitted which does not comply with these terms. 